treatment are basic supportive therapy for those patients. In some cases, surgery is needed and it can involve prognostic features.

Objectives: To analyze prognostic factors related to articular and periarticular infectious diseases.

Methods: Patients hospitalized between January and December 2019 in Rheumatology Service (CHUS) and diagnosed of articular or periarticular infectious disease were enrolled. Demographic, clinical and therapeutic data were collected.

Stata 15.1 was used to perform statistical analysis.

Results: 21 patients diagnosed of articular or periarticular infectious disease were included (138 patients hospitalized in 2019). Mean age was 57,3 years old (SD 18,2) and $76,2 \%$ were males. One cardiovascular risk factor was achieved, at least, by $71,4 \%$ (arterial hypertension the most frequent, followed by hypercholesterolemia and hyperuricemia). Gram positive cocci were identified in $76,2 \%$ of patients (Staphylococcus aureus in $47,6 \%$, only one case oxacillin-resistant). A concomitant rheumatic disease was present in 4 patients (2 suffer from gout). No statistical association was found between this topic and acute-phase reactants (APR), complication rates or days of hospitalization. At hospitalization time, mean values of erythrocyte sedimentation rate (ESR) were 65,6 mm (SD 30,8) and C-reactive protein (CRP) 11,7 mg/dl (SD $7,7)$. Both have a positive association with days of hospitalization $\left(r_{E S R}=0,282\right.$ $\left.r_{C R P}=0,424\right)$ and further, ESR demonstrates a positive correlation with this topic $(\mathrm{Cl} 95 \%-0,009$ to $0,723 \mathrm{p}=0,05569)$. Only 3 patients needed adjuvant surgery (articular infectious diseases with higher APR values). Surgery demonstrates a relationship statistically significant with articular involvement $(t=-3,72 p=0,00)$, higher hospitalization rates $(t=-2,51 p=0,02)$ and complication rates $(\mathrm{Chi} 2=4,67 \mathrm{p}=0,03$ as decease, recurrence, bacteriemia, aftermath or soft tissue abscess).

Conclusion: Gout is the main rheumatic disease associated, in this study, to infectious diseases of musculoskeletal system. As opposed, to suffer a rheumatic disease isn't associate to a bad prognostic (hospitalization or complication rates). Furthermore, this cohort shows us the high prevalence of cardiovascular risk factors related with these diseases (hyperuricemia as predecessor of gout). Surgery intensifies as the main bad prognostic factor regarding days of hospitalization, complication rates and higher APR.

References:

[1] Morbimortality in adult patients with septic arthritis: a three-year hospital-based study. Ferrand J, et al. BMC Infect Dis 2016

[2] Predictors of treatment failure and mortality in native septic arthritis. Maneiro JR, et al. Clin Rheum 2014

Disclosure of Interests: None declared

DOI: 10.1136/annrheumdis-2020-eular.4807

\section{FRI0437 CT GUIDED NEEDLE BIOPSY IN VERTEBRAL OSTEOMYELITIS: RELIABILITY ANALYSIS AND STUDY OF WHICH FACTORS COULD INFLUENCE ON THE RESULT}

J. J. Fragio-Gil ${ }^{1}$, R. Gonzalez Mazario수, E. Grau García ${ }^{1}$, M. De la Rubia Navarro ${ }^{1}$, C. Pávez Perales ${ }^{1}$, S. Leal Rodriguez ${ }^{1}$, J. Ivorra Cortés ${ }^{1}$, L. Gonzalez Puig $^{1}$, R. Negueroles Albuixech ${ }^{1}$, I. Martínez Cordellat ${ }^{1}$, I. Chalmeta Verdejo ${ }^{1}$, J. E. Oller Rodríguez ${ }^{1}$, C. Alcañiz Escandell ${ }^{1}$, E. Vicens Bernabeu ${ }^{1}$, F. M. Ortiz Sanjuan ${ }^{1}$, C. Nájera Herranz ${ }^{1}$, I. Cánovas Olmos ${ }^{1}$, A. J. Cañada Martinez², M. Salavert Lleti ${ }^{3}$, J. A. Román Ivorra ${ }^{1}{ }^{1}$ Hospital Universitario y Politécnico La $\mathrm{Fe}$, Rheumatology, València, Spain; ${ }^{2}$ Instituto de Investigación Sanitaria La Fe de Valencia, Biostatistic, València, Spain; ${ }^{3}$ Hospital Universitario y Politécnico de La Fe, Infectious Disease Unit, València, Spain

Background: Vertebral Osteomyelitis is an infectious disease of the vertebral body, also termed spondylodiscitis if the intervertebral disc is involved (which its avascular). Since the bacteriological characterization is in many times difficult and blood cultures are often negative, a bone biopsy is in most of the cases encouraged.

Objectives: The aim of this study is to analyze which factors could influence on the result of a CT guided biopsy (CTGB) in vertebral spondylodiscitis patients.

Methods: A retrospective observational study was performed including patients diagnosed of spondylodiscitis in a single center who underwent a CTGB. Demographic features and comorbidities, acute phase markers, microbiological results, radiological data, antibiotic exposure, medical complications and the clinical outcomes were also collected for analysis. Standard procedure in our center is performed by Musculoskeletal Specialized Radiologist under local anesthesia and CT control. Abscess sample is collected with a $18 \mathrm{G}$ needle with coaxial technique, trying to obtain at least 3 samples. For discal space, a thicker needle (13.5G-15G) is used. A logistic regression including cofounding factors was performed using $\mathrm{R}$ software.
Results: A total of 86 were included with a mean age of 62.75 (14.98) years old and predominationg male sex (68.60\%). 15 patients (17.44\%) presented any kind of immunosuppression. Clinical data are summarized in Table 1. Blood cultures were positive in $39.71 \%$ and sample culture showed a reliability of $49 \%$. Organism which grew were gram $+(66.67 \%)$, gram $-(12.70 \%)$, mycobacteria $(12.7 \%)$ and fungi $(7.94 \%)$. In only 16 cases $(18.6 \%)$ there was isolated the same organism in blood and on biopsy culture. From admission to procedure, a mean of 6 days was observed. Antibiotic treatment had a median value of 2 days $(0,6)$ and its exposure did not modified the culture positivity (IC 95\% [0.2745.211] $p=0.816$ ). Detailed analysis was performed looking for the influence of the days of exposure, which also failed (IC 95\% [0.939-1.101] $p=0.747$ ). The longer duration of the pain was related to a higher probability of obtaining a negative result on the biopsy (IC 95\% [1.004-1.035] $p=0.026$ ) (graphic 1). Neither fever $(p=0.303)$ or higher CRP (IC 95\% [0.992-1.006] $p=0.761)$ value modified the culture result.

Table 1. Demographic and clinical characteristics.

\begin{tabular}{lcc}
\hline & $\mathrm{N}=86$ & $\%$ \\
\hline Clinical history & & \\
High blood pressure & 42 & 48.84 \\
Diabetes Mellitus & 19 & 22.09 \\
Liver cirrhosis & 16 & 18.60 \\
Chronic kidney failure & 13 & 15.12 \\
Active Systemic Malignancy* & 2 & 2.33 \\
Rheumatoid arthritis* & 3 & 3.49 \\
Spondyloarthritis* & 1 & 1.16 \\
HIV infection* & 4 & 4.65 \\
Solid organ transplant receptor* & 3 & $3.49 \%$ \\
Systemic Amyloidosis* & 1 & 1.16 \\
Splenectomy* & 2 & 2.33 \\
Previous spine pathology & 50 & 58.14 \\
Underlying/associated endocarditis & 2 & $2.33 \%$ \\
& &
\end{tabular}

${ }^{*}$ Considered as immunosuppressed patients

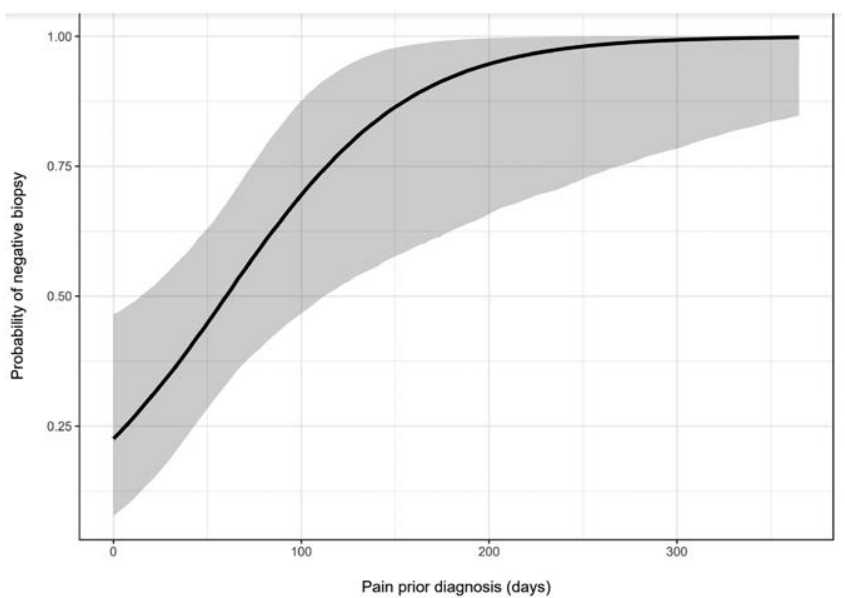

Conclusion: Even in cases under antibiotic treatment, CTGB displays an acceptable reliability. The longer the length of painful period before diagnosis was related to a higher chance of obtaining a negative result on culture. This result could be explained by a greater aggressiveness of pyogenic organisms that perhaps congregate in the lesser time span instead of non-pyogenic agents, that could deliver in more silent infection.

References:

[1] IDSA Clinical Practice Guidelines for the Diagnosis and Treatment of Native Vertebral Osteomyelitis in Adults

Disclosure of Interests: None declared

DOI: 10.1136/annrheumdis-2020-eular.6383

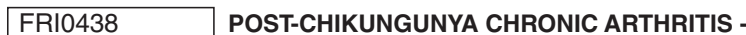 SHARING OF BANGLADESH EXPERIENCE OVER ONE} YEAR FOLLOW UP OF 60 PATIENTS

S. Hossain ${ }^{1}$, M. Choudhury², M. A. Islam², M. M. Hassan², S. Yeasmin²,

F. Hossain ${ }^{3} .{ }^{1}$ Bangabandhu Sheikh Mujib Medical University (BSMMU), Department of Rheumatology, Dhaka, Bangladesh; ${ }^{1}$ Bangabandhu Sheikh Mujib Medical University (BSMMU), Department of Rheumatology, Dhaka, Bangladesh; ${ }^{3}$ Freelance Researcher, Dhaka, Bangladesh 
Background: Chikungunya virus (CHIKV) infection is an emerging disease which is responsible for several epidemics around the world ${ }^{1}$. Systematic review and meta-analysis had shown that approximately $25 \%$ of cases of Chikungunya($\mathrm{CHIK}$ ) would develop CHIK-Chronic Inflammatory Rheumatism and $14 \%$ would develop persistent arthritis (or spondylitis) ${ }^{2}$.

Objectives: To describe the frequency of the clinical patterns of chronic arthritis \& to characterize the clinical symptoms in a Bangladeshi cohort of CHIK patients 12 months post-infection.

Methods: In 2017, a Chikungunya outbreak occurred in Dhaka, Bangladesh, during which a prospective cohort of $\mathrm{CHIK}$ patients with confirmed diagnosis was constituted. A longitudinal follow up of 60 patients from an initial cohort of 142 patients, attending the out-patient department of Rheumatology, BSMMU, was done. Patients having arthritis/ arthralgia or both lasting more than 3 months were considered as chronic cases. Their baseline and follow-up symptoms at $3 \mathrm{~m}, 6 \mathrm{~m}$ and $12 \mathrm{months}$ were evaluated. Functional status was assessed with the Bengali Version Health Assessment Questionnaire (HAQ).

Results: Of the initial 142 patients enrolled in the study, 135(95.1\%) had CHIKV-lgM and $29(20.4 \%)$ had $\mathrm{lgG}$ positive. Patients that followed up in-person were predominantly adult (age $43.73 \pm 11.09$ years) and female $34(56.7 \%)$. The majority of the patients $35(58.3 \%)$ had undifferentiated arthritis. After three months, $8(16.3 \%)$ had oligoarthralgia, $26(53.1 \%)$ had polyarthralgia and $8(16.3 \%)$ had polyarthralgia with oligoarthritis. At the end of one year, $13(21.7 \%)$ patients underwent complete remission. Among the 47 patients, 21 had joint involvement where $11(52.4 \%)$ had polyarthralgia, $5(23.8 \%)$ had polyarthralgia with oligoarthritis, $5(23 \%)$ had oligoarthralgia and $4(19 \%)$ had monoarthralgia. Among the 47 patients, mild, moderate and severe functional disability was present in $89.4 \%, 6.4 \%$ and $4.3 \%$ patients respectively. Conclusion: After one year of follow up, one-third of the patients remained symptomatic. Polyarthralgia was the predominant clinical feature. Mild functional disability was also observed in a significant number of patients.

References:

[1] Ali Ou Alla S, Combe B. Arthritis after infection with Chikungunya virus. Best Pract Res Clin Rheumatol. 2011;25(3):337-46.

[2] Rodríguez-Morales AJ et al. Prevalence of Post-Chikungunya Infection Chronic Inflammatory Arthritis: A Systematic Review and Meta-Analysis. Arthritis. Care. Res. 68(12), 1849-1858 (2016).

Table 1. Socio-demographic and clinical pattern of patients with Chronic CHK arthritis $(n=60)$

\begin{tabular}{lc}
\hline Variables & $\mathrm{n}(\%)$ \\
\hline Age (in years) & \\
Mean \pm SD & $43.73 \pm 11.1$ \\
Sex & $34(56.7)$ \\
Female & $26(43.3)$ \\
Male & \\
First joint involvement & $34(56.7)$ \\
Generalized & $12(20.0)$ \\
Ankle & $6(10.0)$ \\
Knee & $5(8.3)$ \\
Wrist & $3(5.0)$ \\
Others (MTP, shoulder, neck and axial plane) & \\
Clinical pattern & $35(58.3)$ \\
Undifferentiated & $10(16.7)$ \\
Spondyloarthritis & $7(11.7)$ \\
Rheumatoid Arthritis & $6(10.2)$ \\
Pre-existing Spondyloarthritis & $1(1.7)$ \\
Pre-existing Rheumatoid Arthritis & $1(1.7)$ \\
Pre-existing Osteo-arthritis Arthritis &
\end{tabular}

Table 2. Joint involvement of patients with Chronic CHIK arthritis

\begin{tabular}{|c|c|c|c|}
\hline \multirow[t]{2}{*}{ Joint characteristics } & \multirow{2}{*}{$\frac{3 m(n=49)}{f(\%)}$} & \multirow{2}{*}{$\frac{6 m(n=36)}{f(\%)}$} & \multirow{2}{*}{$\frac{1 \mathrm{yr}(n=21)}{f(\%)}$} \\
\hline & & & \\
\hline \multicolumn{4}{|c|}{ Type of involvement in persistent pain } \\
\hline Joint only & $33(55.0)$ & $28(46.7)$ & 20 (33.3) \\
\hline $\begin{array}{l}\text { Both joint \& soft-tissue } \\
\text { Joint involvement }\end{array}$ & $16(26.7)$ & $8(13.3)$ & $1(1.7)$ \\
\hline Monoarthralgia & $1(2.0)$ & $5(13.9)$ & $4(19.0)$ \\
\hline Oligoarthralgia & $8(16.3)$ & $6(16.7)$ & $5(23.0)$ \\
\hline Polyarthralgia & $26(53.1)$ & $18(50.0)$ & $11(52.4)$ \\
\hline Monoarthritis & $1(2.0)$ & $0(0.0)$ & $0(0.0)$ \\
\hline Oligoarthritis & $6(12.2)$ & $3(8.3)$ & $0(0.0)$ \\
\hline Polyarthritis & $7(14.3)$ & $9(11.1)$ & $1(4.8)$ \\
\hline Oligoarthralgia \& monoarthritis & $1(2.0)$ & $0(0.0)$ & $0(0.0)$ \\
\hline Polyarthralgia \& monoarthritis & $0(0.0)$ & $1(2.8)$ & $0(0.0)$ \\
\hline Polyarthralgia \& oligoarthritis & $8(16.3)$ & $4(11.1)$ & $5(23.8)$ \\
\hline Polyarthralgia \& Polyarthritis & $1(2.0)$ & $0(0.0)$ & $0(0)$ \\
\hline
\end{tabular}

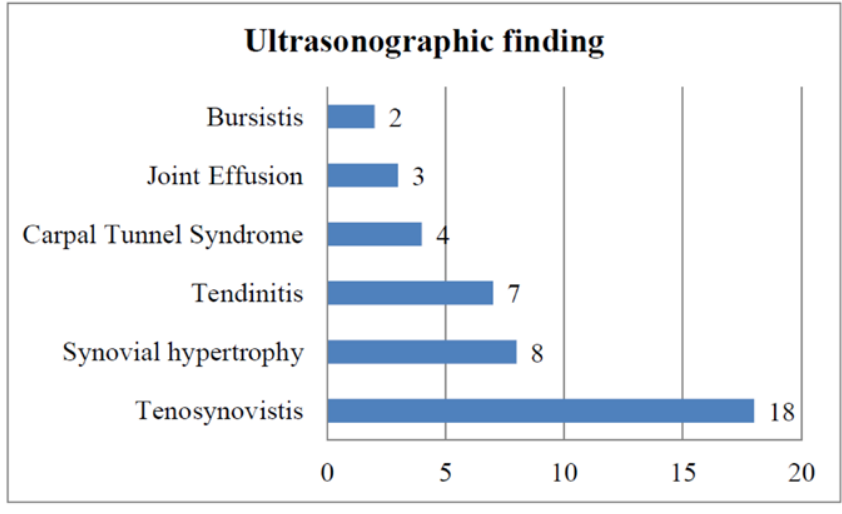

Figure 1: Distribution of patients by ultrasonographic findings $(n=60)$

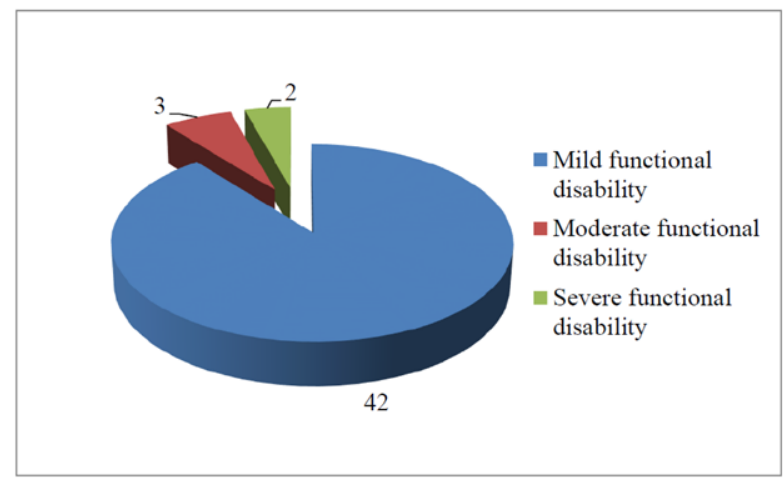

Figure 2: Distribution of patients by functional disability after 1 year $(n=47)$

Disclosure of Interests: None declared

DOI: 10.1136/annrheumdis-2020-eular.6101

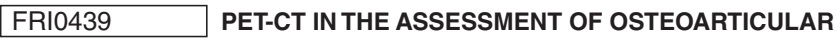 INFECTIONS ASSOCIATED WITH INFECTIVE ENDOCARDITIS}

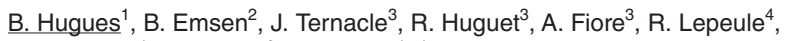
X. Chevalier ${ }^{1}$, M. Abulizi ${ }^{2}$, F. Eymard ${ }^{1}{ }^{1}$ Hôpital Henri Mondor, Rheumatology, Créteil, France; ${ }^{2}$ Hôpital Henri Mondor, Nuclear Medecine, Créteil, France; ${ }^{3}$ Hôpital Henri Mondor, Cardiology, Créteil, France; ${ }^{4}$ Hôpital Henri Mondor, Infectiology, Créteil, France

Background: Five to eleven percent of infective endocarditis (IE) are associated with a musculoskeletal infection. Thanks to its good sensitivity, the use of PET-CT in this pathology makes it possible to confirm the diagnosis by seeking valvular hypermetabolism but also by mapping distant septic foci.

Objectives: The main objective of our study was to assess the prevalence of osteoarticular fixation (OAF) with PET-CT, symptomatic or not, in patients with IE. The secondary objectives were to determine predictive factors for osteoarticular infections such as the type of valve damage (native or prosthetic) and germ.

Methods: This study was carried out on the basis of a prospective cohort of patients admitted in the department of cardiology in Henri Mondor Hospital for IE suspicion between August 2015 and July 2019. Demographic, clinical, bacteriological, imaging and therapeutic data have been collected. Patients matching Duke's modified criteria according to ESC 2015 (Duke + IE) and / or a cardiac fixation according to standard whole-body PET-CT were included in the analysis. All of the PET-CT scans were reviewed by a nuclear medicine specialist to confirm whether or not there was a heart condition and to look for septic-looking OAF.

Results: From this cohort, we included 90 IE Duke + patients and 42 patients with cardiac PET-CT fixation (including 31 IE Duke +). In the IE Duke + group, we found OAF in 18 patients (20\%), 39\% of whom were asymptomatic. There were 9 spondylodiscitis ( 4 on the cervical level, 7 on the thoracic level, and 2 on the lumbar level), 5 glenohumeral arthritis, 2 coxofemoral arthritis, 1 sternoclavicular arthritis and 1 sacroiliac arthritis. The IE affected the aortic valve in $50 \%$ of the cases and the mitral valve in $22 \%$. In other cases, the infection involved the internal automatic defibrillator (ICD) or the pacemaker. The valves were prosthetic in $73 \%$ of the cases. The bacterial ecology was mainly represented by 